\title{
On Contradictory Christology: Preliminary Remarks, Notation and Terminology
}

\author{
Jc Beall \\ University of Connecticut
}

\begin{abstract}
The following are some preliminary remarks that will set the stage for my individual replies to Timothy Pawl, Thomas McCall, A. J. Cotnoir, and Sara L. Uckelman's responses to my paper 'Christ - A Contradiction'. In that paper I advance and defend a contradictory Christology which solves the fundamental 'problem' of Christology by holding that Christ is a contradictory being: it is true that Christ is mutable and it is false that Christ is mutable; it is true that Christ is immutable and it is false that Christ is immutable; and so on for other fundamental-problem properties. Familiarity with 'Christ - A Contradiction' (and also with background discussion including, e.g., Richard Cross's discussions of 'the fundamental philosophical problem') is assumed.
\end{abstract}

\section{Aim of these preliminary remarks}

The aim of these preliminary remarks is to review or otherwise clarify salient terminology involved in my target position, namely, Contradictory Christology (or a contradictory theology in general). Not all (or even most) issues are touched on in these preliminaries; only issues that run constant through the symposiasts' papers are reviewed here.

Gratitude. While I acknowledge each individual symposiast in my respective replies I want to express my deepest gratitude to all of them - and to the editors - for their work on this symposium. The care and attention focused on my nascent contradictory theology is a gift whose value to the project is beyond what I can adequately express, and also a gift that has humbled me more than I will publicly express. What I can and will say here, at the start, is just this: thank you - to the editors, to the participants in Notre Dame's CPR seminars, and above all to the symposiasts. Thank you.

\section{Contradictory Christology: brief recap}

Contradictory Christology, as advanced in 'Christ - A Contradiction', takes the strong appearance of Christ's contradictory being - the appearance at the root of what Cross

Journal of Analytic Theology, Vol. 7, June 2019

10.12978/jat.2019-7.352828273037

(c) 2019 Jc Beall • (C) 2019 Journal of Analytic Theology 
coined 'the fundamental problem' of Christology - to be veridical: it is true that Christ is mutable and it is false that Christ is mutable. (And there are many other fundamental-problem contradictions true of Christ.) Contradictory Christology is so called because it is a contradictory theory; it contains contradictions.

On the mainstream view of logical consequence, where logic is the foundational entailment relation involved in all true theories (and in that way logic is 'universal'), a contradiction in any theory 'explodes' the theory into its so-called trivial one - the theory according to which all sentences (in the language of the theory) are true. The trivial christology is absurd by all lights; and it is plainly untrue (since at least some of the sentences in the language are untrue). Any viable contradictory christology (theology, generally) rejects the mainstream view of logical consequence, not in its universal application but rather in its claims about the explosiveness of contradictions. ${ }^{1}$

\section{Logical versus theory-specific consequences}

An issue in the foreground of various papers is the difference between logical consequence and theory-specific - in the pressing case, theology-specific consequence relations. Logical consequence is universal; it's part of every consequence relation involved in any true (and complete-as-possible) theory. ${ }^{2}$ useful.

A review (and elaboration) of terminology from 'Christ - A Contradiction' is

\subsection{Explosive sentences}

Let us say that a sentence $A$ explodes according to a consequence relation $\vdash_{T}$ iff every sentence (in the language of theory T) is a consequence of $A$, that is, if $A \vdash_{T} B$ (read ' $A T$-entails $B$ ' or ' $A \therefore B$ ' is $T$-valid) for every sentence $B$ in the relevant language.

\footnotetext{
${ }^{1}$ Actually, Dahms, who deserves credit for pointing to a different account of logic to accommodate true theological theory, seems to have rejected the standard account not on its view of contradictions exploding but rather on the universal applicability of logic (see Dahms 1978). As per 'Christ - A Contradiction' I firmly reject such a position. From my perspective, to reject the constraints of logic on true theology is to reject that theology is a truth-seeking discipline.

2 Technically, the logical vocabulary (here taken to be the standard stock of first-order vocabulary sans identity, and whose material conditional is defined per usual via negation and disjunction) is involved in all true theories, and it is the vocabulary in terms of which 'logical forms' - the argument forms on which logical consequence has any say - are defined. Consequence relations of our true theories 'extend' logical consequence in the sense that if $X \therefore A$ is logically valid then it's valid according to the consequence relation of the true theory too. (Important: that $X \therefore A$ is logically invalid is compatible with its being valid according to the consequence relation for some true theory. Much more on this below.)
} 


\subsection{Formal contradictions}

Now, let $\wedge$ be logical conjunction and $\neg$ logical negation (i.e., logic's unary falsity operator). Call any sentence of the form

$$
A \wedge \neg A
$$

a formal contradiction - for present purposes, a contradiction (simpliciter). ${ }^{3} \mathrm{~A}$ formal contradiction is literally read (using logic's unary truth operator):

It is true that.... and it is false that...

In the case of a sentence like 'Christ is mutable' the contradiction is:

It is true that Christ is mutable and it is false that Christ is mutable.

Of course, the word 'not' is often employed as a stand-in for logical negation (i.e., for logic's falsity operator), ${ }^{4}$ in which case, where 'not' is so used, the given contradiction is:

It is true that Christ is mutable and Christ is not mutable.

\subsection{The mainstream view of logic and contradictions}

According to the standard (mainstream) view of logical consequence, contradictions are explosive sentences according to logic (i.e., according to logical consequence), that is, where $\vdash$ (unsubscripted because part of every true theory) is logical consequence,

$$
A \wedge \neg A \vdash B
$$

(abbreviated: $! A \vdash B$ ). And since this is a logical entailment, it's an entailment according to the consequence relation of every true theory. So goes the standard view.

\subsection{Contradictions and Contradictory Christology}

According to Contradictory Christology (as advanced in 'Christ - A Contradiction') the standard account of logical consequence goes too far; the standard account mistakes common consequences involved in many true theories with logical consequences. The correct view of logical consequence, according to Contradictory Christology, is

\footnotetext{
${ }^{3}$ Notation: for both shorthand and ease on the eye I often use '! $A$ ' as an abbreviation for ' $A \wedge \neg A$ '.

${ }^{4}$ Often so employed; not always. (And sometimes the usage of 'not' is a theory-specific or highly theoryladen technical term used to comment on logical negation - as in, perhaps, claims like 'both the sentence and its logical negation are not true', but I set these technical tangents aside here.)
} 
one according to which contradictions are not explosive according to logic; 5 hence, contradictions need not be explosive in all true theories. But they may be explosive in some. Contradictions may be - and, in fact, I think that they are - explosive according to many of our true theories (e.g., arithmetic, biology, and others); however, many is not all. And according to Contradictory Christology contradictions are not explosive in the true theology.

\subsection{Contradictions and theory-specific absurdities}

How can contradictions be explosive according to some true theories (in particular, their consequence relations) while they are not explosive according to logic - the universal consequence relation common to all true theories? The answer is that logical consequence is an absence-of-counterexample relation: a pair of sentences $A \therefore$ $B$ (or, generally, a pair from a set $X$ to sentence $B$ ) is logically valid iff logic (-al consequence) sees no counterexample to the pair, where a counterexample is a possibility in which $A$ (or everything in set $X$ ) is true but $B$ fails to be true. ${ }^{6}$ But now suppose that a theory restricts the wide space of logical possibilities to some properly smaller space of what the theory treats as theoretical possibilities, and in turn the theory's consequence relation looks only at the theoretical possibilities as would-be counterexamples to pairs $A \therefore B$ (or $X \therefore B$ ) in the language of the theory. In this case, a theory might well ignore all logical possibilities in which a contradiction $! A$ is true - that is, the theory might ignore all glutty logical possibilities, treating them as theoretically impossible. In this case, where $\vdash_{T}$ is the theory's consequence relation, contradictions are explosive according to the theory:

$$
\text { ! } A \vdash_{T} B
$$

even though, as above, logic itself (viz., $\vdash$, which is a part of $\vdash_{T}$ ) doesn't validate the would-be entailment.

Let us say that a sentence $A$ is absurd according to theory $T$ iff $A$ is explosive according to T. As above, Contradictory Christology maintains that contradictions aren't absurd according to all theories, though they may be (and, I say, are) absurd according to some - for example, arithmetic, biology, probably others.

Important to understand is that true theories can (perhaps often do) treat some non-contradictions (i.e., sentences not of the form $! A$ ) as absurd - even if they do not treat contradictions as absurd. Witness: take the true theology. On an orthodox account (or at least a Chalcedon-constrained account) the following two sentences are jointly absurd:

\footnotetext{
${ }^{5}$ The correct account of logic, I believe, is so-called first-degree entailment (FDE), so called in Anderson and Belnap 1975, and sufficiently presented, for present purposes, in the symposium's target paper (viz., 'Christ - A Contradiction'). When I talk about logic (i.e., logical consequence) I am talking about logical consequence per the FDE account.

${ }^{6}$ Worth keeping in mind too is that logical validity looks only at the logical vocabulary; it treats everything except logical vocabulary as, in effect, a neutral variable (so to speak).
} 
1. It's false that Christ is divine.

2. It's false that Christ is human.

These are jointly (just) false in all (non-trivial) models of the true theory (according to a suitably Chalcedon-constrained account); there are no (non-trivial) models of the theory in which both of the given sentences are true. ${ }^{7}$ If these sentences are jointly (just) false in all models of the given theory, their logical conjunction is absurd: it is explosive according to the theory inasmuch as there is no counterexample from the conjunction of (1) and (2) to an arbitrary sentence. (A counterexample from the conjunction of those sentences to an arbitrary sentence requires a possibility, recognized by the theory, in which the given conjunction is at least true.)

Whether you think that (1) or (2) or even their logical conjunction is an example of an absurd sentence in the true theology is not as pressing as whether there can be absurd sentences in the true theology which are not contradictions. I think that (1) and (2) are good examples but others can be offered, including:

3. God is Zeus.

4. It's false that there exists a god.

And there are others.

\footnotetext{
${ }^{7}$ The trivial model of a theory is the (unique) model in which all sentences in the language of the theory are true. Theories need not involve a trivial model, but they make no real difference to a theory's consequence relation. (The models are 'allowed' as far as logic - that is, logical consequence - itself is concerned.)
} 


\section{Bibliography}

Anderson, Alan Ross and Nuel D. Belnap. 1975. Entailment: The Logic of Relevance and Necessity. Vol. 1. Princeton: Princeton University Press.

Anderson, Alan Ross, Nuel D. Belnap, and J. Michael Dunn. 1992. Entailment: The Logic of Relevance and Necessity. Vol 2. Princetion: Princeton University Press.

Dahms, John V. 1978. "How reliable is logic?" Journal of the Evangelical Theological Society, 21: 369-380. 